\title{
Contributions to The Bryophyte Flora of Hayrat District (Trabzon - Turkey)
}

\author{
Öznur ÖZEN ${ }^{1}$ (D), Hüseyin ERATA² ${ }^{\text {ID }}$, Nevzat BATAN*3 ${ }^{\text {ID }}$, Mevlüt ALATAŞ ${ }^{4}$ \\ ${ }^{1}$ Karadeniz Technical University, Department of Biology, Faculty of Science, Trabzon, TURKEY \\ ${ }^{2}$ Çanakkale Onsekiz Mart University, Bayramiç Vocational School, Çanakkale, TURKEY \\ ${ }^{3}$ Karadeniz Technical University, Maçka Vocational School, Trabzon, TURKEY \\ ${ }^{4}$ Munzur University, Faculty of Engineering / Bioengineering, Tunceli, TURKEY
}

$\begin{array}{lll}\text { Received: 03.05.2019 } & \text { Revised: 05.07.2019 } & \text { Accepted: 13.07.2019 }\end{array}$

\begin{abstract}
As a result of bryological excursion carried out in the Hayrat district of Trabzon province, a total of 99 bryophyte taxa, including 18 liverworts and 81 mosses, were determined from the samples collected from 6 different localities. All of these taxa were reported for the first time from Hayrat district. Brachythecium capillaceum (F.Weber \& D.Mohr) Giacom. was recorded as a new record for Trabzon province and the second record for A4 grid-square.
\end{abstract}

Key words: Biodiversity, Bryophyte, Flora, Hayrat District, Turkey

\section{Hayrat İlçesi (Trabzon-Türkiye) Briyofit Florasına Katkılar}

\begin{abstract}
$\ddot{O} z$
Trabzon ilinin Hayrat ilçesi'nde yapılan arazi çalışmaları sonucunda, 6 farklı lokaliteden toplanan örneklerden 18 Ciğerotu ve 81 Yapraklı karayosunu olmak üzere toplam 99 briyofit taksonu tespit edilmiştir. Bu taksonların hepsi Hayrat ilçesi için ilk kez kaydedilmiştir. Brachythecium capillaceum (F.Weber \& D.Mohr) Giacom. Trabzon ili için yeni kayıt, A4 karesi için ikinci kayıt olarak rapor edilmiştir.
\end{abstract}

Anahtar kelimeler: Biyoçeşitlilik, Briyofit, Flora, Hayrat İlçesi, Türkiye.

\footnotetext{
* Corresponding author: nevzatbatan@gmail.com (C) 2019 All rights reserved / Tüm haklarl saklıdır.

To cite this article: Özen Ö. Erata E. Batan N. Alataş M. 2019. Contributions to The Bryophyte Flora of Hayrat District (Trabzon - Turkey). Anatolian Bryology. 5:2, 107-113.

(c) (1) () T) This work is licensed under a Creative Commons Attribution-NonCommercial 4.0 International License.
} 


\section{Introduction}

Hayrat is situated in the Euro-Siberian floristic region and is located in the A4 square of Turkey according to the grid system of Henderson (Henderson, 1961). The study area is in Trabzon province in the Black Sea region of Turkey.

Hayrat district is surrounded by Of district in the north, Çaykara district and Bayburt province in the south, Rize province in the east, Of, Çaykara and Dernekpazar1 districts in the west (Figure 1). Hayrat, which is $12 \mathrm{~km}$ away from the Black Sea coastline, has an area of $170 \mathrm{~km}^{2}$ and an average altitude of $180 \mathrm{~m}$. The research area has warm and rainy in summers and cool and rainy Black Sea climate in winter, and no dry season (Akman, 1999).

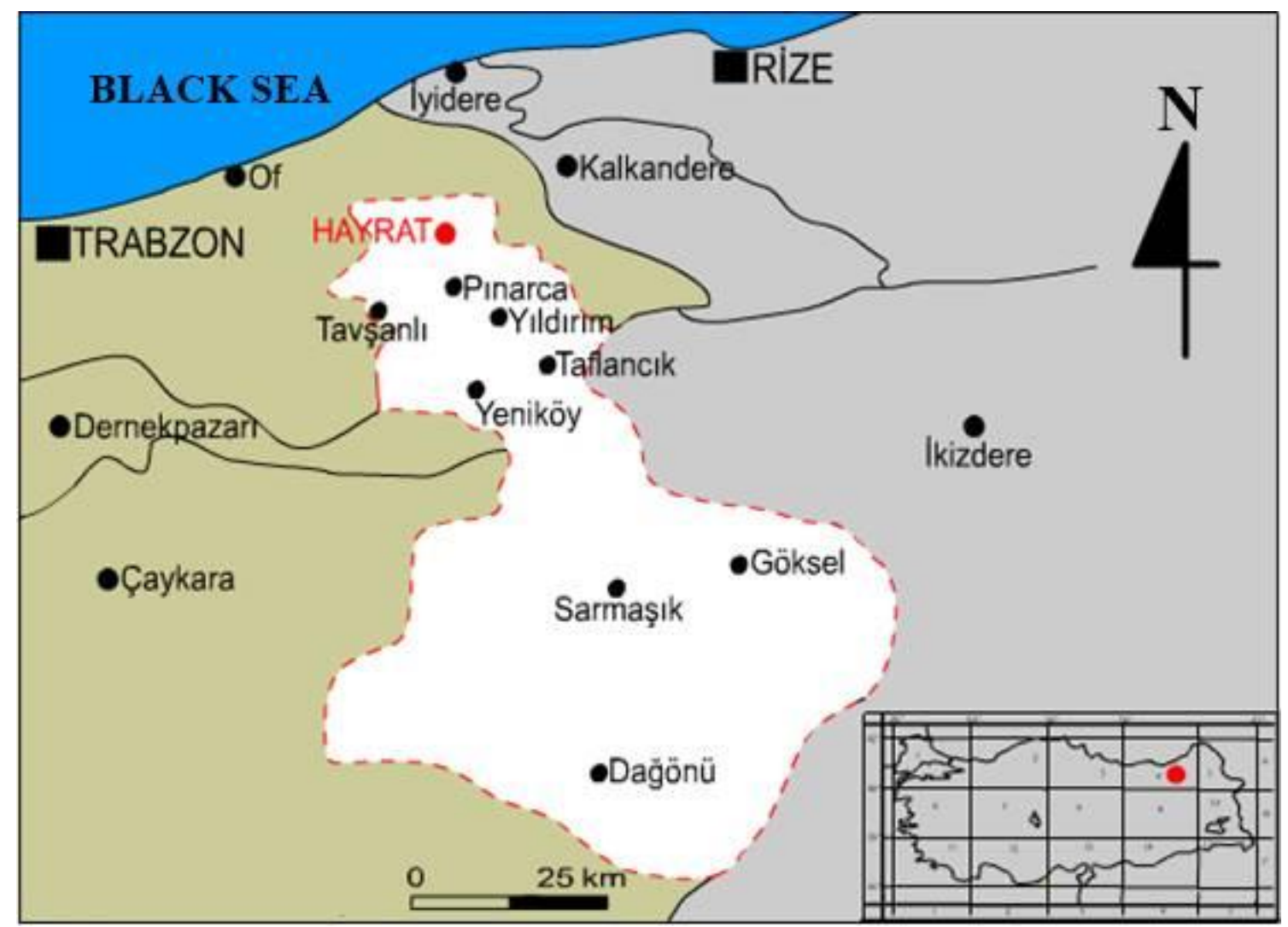

Figure 1. Map of the Study area

Although the diversity of vascular plants (Atay et al., 2009) and knowledge about vegetation (Vural, 1996) in different parts of Turkey are well known, the bryophyte flora of many regions of Turkey is still poorly documented or completely unknown. But in recent years, there has been carried out bryofloristic studies in Trabzon province (Gökler, 1998; Papp, 2004; Townsend, 2005; Lara et al., 2010; Batan and Özdemir, 2011; Kirmaci et. al., 2012; Batan et al., 2013; Kırmac1 and Kürschner, 2013; Batan and Özdemir, 2013; Özdemir and Batan, 2017a). The aim of the present study is to contribute to the moss flora of Trabzon province (Hayrat districts) and the moss flora of Turkey. In addition, since there is no previous study in Hayrat district, the samples of liverworts and mosses collected from various regions are new to this region.

\section{Materials and Methods}

The bryophyte samples were collected in 6 different localities from the Hayrat district in Trabzon. The bryophyte samples were examined with Carl Zeiss Stemi 2000-C stereo-microscope and Carl Zeiss Axio İmager A2 light microscope. Identifications were determined by consulting various floras and keys (Nyholm, 1986, 1989, 1993, 1998; Blom, 1996; Smith, 1996, 2004; Paton, 1999; Pedrotti, 2001, 2006; Frey et al., 2006; Guerra et al., 2006; Brugués et al., 2007; Kürschner and Frey, 2011).

For each taxon, localities and substrate were given to avoid repetition in the floristic list, but the same plants collected from different localities were indicated (Loc. 1, 2, 3,... etc.). First time record for Trabzon is indicated with (\#), second time record for an A4 grid-square is indicated 
with (+), the taxa recorded from Trabzon for the second time are indicated with $\left(^{*}\right)$ in the bryofloristic list. Nomenclature of the species follows Söderström et al., (2016) for liverworts and Ros et al., (2013) for mosses. The situation of bryophyte taxa was appraised by reviewing related literature for the Trabzon (Özdemir and Batan, 2017a,b). The status of bryophyte taxa was evaluated by reviewing related literature for the A4 square (Abay et al., 2016; Özdemir and Batan, 2017a, b). Vouchers are deposited in the Biology Department, Faculty of Science, Karadeniz Technical University, Turkey (KTUB).

Abbreviations in the floristic list: Station no: (1, $2,3, \ldots),(\mathrm{S})$ : on soil, $(\mathrm{R})$ : on rock, $(\mathrm{SM})$ : submerged, (DTT): on dead tree trunk, (TB): on tree body, (WS): on wet soil, (WR): on wet rock and (NS): near stream and name "Özen" with number means the herbarium number.

\subsection{Locations of the collected bryophyte specimens}

1. Trabzon province, Hayrat district, between Pınarca Village and Yildırımlar Village (Çağlayan Village 3) $40^{\circ} 51^{\prime} 49^{\prime \prime} \mathrm{N}, 40^{\circ} 22^{\prime} 25^{\prime \prime}$ E, 520-550 m.

2. Trabzon province, Hayrat district, Taflancık Village-1, 40 49' 43" N, 40²4' 17" E, 510 m.

3. Trabzon province, Hayrat district, Taflancik Village-2 (on the way to highland-1) $40^{\circ} 50^{\prime} 38^{\prime \prime}$ $\mathrm{N}, 40^{\circ} 22^{\prime} 58^{\prime \prime} \mathrm{E}, 820-830 \mathrm{~m}$.

4. Trabzon province, Hayrat district, between Hayrat and Pınarca Village (Çağlayan Village-2), $40^{\circ} 52^{\prime} 45,8^{\prime \prime} \mathrm{N}, 40^{\circ} 21^{\prime} 48^{\prime \prime} \mathrm{E}, 360 \mathrm{~m}$.

5. Trabzon province, Hayrat district, between Hayrat and Pınarönü Village (Çağlayan Village1), $40^{\circ} 53^{\prime} 07,6^{\prime \prime} \mathrm{N}, 40^{\circ} 21^{\prime} 32,9^{\prime \prime} \mathrm{E}, 205 \mathrm{~m}$.

6. Trabzon province, Hayrat district, Taflancik Village -2, $43^{\circ} 49^{\prime} 43^{\prime \prime} \mathrm{N}, 40^{\circ} 24^{\prime} 18^{\prime \prime} \mathrm{E}, 500 \mathrm{~m}$.

\section{Findings}

Bryofloristic List

Liverworts (Marchantiophyta)

Conocephaleaceae Müll. Frib. Ex Grolle

Conocephalum conicum (L.) Dumort. - Loc.: 1WR-Özen 1; 2-WS-Özen 2; 3-WS-Özen 3; 5WS-Özen 4.

Pelliaceae H. Klinggr.

Pellia endiviifolia (Dicks.) Dumort. - Loc.: 1WS-Özen 5; 2-WS-Özen 6; 4- WS-Özen 7; 6WS-Özen 8.

P. epiphylla (L.) Corda - Loc.: 3-WS-Özen 9.

Calypogeiaceae Arnell

Calypogeia arguta Nees et Mont. - Loc.: 3-SÖzen 10, R-Özen 11.
Calypogeia fissa (L.) Raddi- Loc.: 1-WS-Özen 12; 4-WS-Özen 13; 5-WS-Özen 14; 6-S-Özen 15.

Scapaniaceae Mig.

Scapania compacta (Roth) Dumort. - Loc.: 6-SÖzen 16, R-Özen 17.

S. nemorea (L.) Grolle - Loc.: 2-WS-Özen 18; 4WR-Özen 19.

Diplophyllum albicans (L.) Dumort. - Loc.: 1-SÖzen 20; 2-R-Özen 21; 6-S-Özen 22, R-Özen 23.

D. taxifolium (Wahlenb) Dumort. - Loc.: 4-WSÖzen 24, R-Özen 25.

Arnelliaceae Nakai

*+Southbya tophacea (Spruce) Spruce- Loc.: 1S-Özen 26; 5-R-Özen 27.

Plagiochilaceae Müll. Frib.

Pedinophyllum interruptum (Nees) - Loc.: 5WS- Özen 28, WR-Özen 29.

Plagiochila asplenioides (L. emend. Taylor) Dumort. - Loc.: 5-S- Özen 30, R-Özen 31.

$P$. porelloides (Torrey ex Nees) Lindenb- Loc.: 2-S- Özen 32, R-Özen 33.

Radulaceae Müll. Frib.

Radula complanata (L.) Dumort. - Loc.: 5-RÖzen 34.

Frullaniaceae Lorch

Frullania fragilifolia (Taylor) Gottsche, Lindenb. \& Nees. - Loc.: 5-DDT-Özen 35.

F. tamarisci (L.) Dumort. - Loc.: 2-R-Özen 36; 6-R-Özen 37.

Jubulaceae H. Klinggr.

Jubula hutchinsiae (Hook.) Dumort. subsp. caucasica Konstant. \& Vilnet - Loc.: 5-NSÖzen 38, WR-Özen 39.

Metzgeriaceae $\mathrm{H}$. Klinggr.

Metzgeria furcata (L.) Dumort. - Loc.: 1-RÖzen 40; 5-TB-Özen 41.

\section{Mosses (Bryophyta)}

Polytrichaceae Schwagr.

Atrichum undulatum (Hedw.) P. Beauv. - Loc.: 1-S-Özen 42; 2-S-Özen 43; 4-WS-Özen 44; 5-SÖzen 45; 6-WS-Özen 46.

Pogonatum aloides (Hedw.) P. Beauv. - Loc.: 6S-Özen 47.

P. urnigerum (Hedw.) P.Beauv. - Loc.: 2-SÖzen 48, R-Özen 49.

Polytrichum commune Hedw. - Loc.: 4-S-Özen 50; 6-S-Özen 51.

P. formosum Hedw. - Loc.: 1-S-Özen 52; 2-SÖzen 53; 6-S-Özen 54.

Grimmiaceae Arn.

Racomitrium aquaticum (Brid. ex Schrad.) Brid. - Loc.: 6-R-Özen 55.

Schistidium papillosum Culm. - Loc.: 3-R-Özen 56.

S. trichodon (Brid.) Poelt- Loc.: R-2-Özen 57. 
Dicranellaceae M. Stech

Dicranella heteromalla (Hedw.) Schimp. - Loc.: 1-S-Özen 58; 2-S-Özen 59; 4-S-Özen 60.

Rhabdoweisiaceae Limpr.

Dichodontium pellucidum (Hedw.) Schimp. .Loc.: 2-S-Özen 61; 3-S-Özen 62; 5-S-Özen 63.

Dicranaceae Schimp.

Dicranum scoparium Hedw. - Loc.: 3-R-Özen 64, TB-Özen 65.

Leucobryaceae Schimp.

*Campylopus brevipilus Bruch \& Schimp. Loc.: 3-WS-Özen 66.

${ }^{*}$ C. flexuosus (Hedw.) Brid.- Loc.: 4-WS-Özen 67; 6-WR-Özen 68.

C. fragilis (Brid.) Bruch \& Schimp- Loc.: 1WS-Özen 69; 4-WS-Özen 70.

${ }^{*}$ C. pyriformis (Schultz) Brid. - Loc.: 6-S-Özen 71.

C. subulatus Schimp. ex Milde. - Loc.: 3-S-Özen 72.

Leucobryum glaucum (Hedw.) Ångstr - Loc.: 1S-Özen 73; 2-S-Özen 74.

L. juniperoideum (Brid.) Müll. Hal. - Loc.: 5-SÖzen 75; 6-WS-Özen 76.

Fissidentaceae Schimp.

Fissidens dubius P. Beauv. - Loc.: 1-WS-Özen 77; 2-WR-Özen 78; 3-WS-Özen 79; 5-WS-Özen 80.

Pottiaceae Schimp.

Barbula unguiculata Hedw. - Loc.: 5-S-Özen 81.

*Bryoerythrophyllum ferruginascens (Stirt.) Giacom. - Loc.: 2-WS-Özen 82; 5-WR-Özen 83.

Gymnostomum aeruginosum Sm. - Loc.: 2-WSÖzen 84; 5-WS-Özen 85.

Tortella squarrosa (Brid.) Limpr. - Loc.: 6-SÖzen 86, R-Özen 87.

T. tortuosa (Hedw.) Limpr. - Loc.: 2-S-Özen 88; 3-R-Özen 89

Trichostomum brachydontium Bruch - Loc.: 2-SÖzen 90

Weissia controversa Hedw. - Loc.: 3-S-Özen 91.

Bartramiaceae Schwagr.

Bartramia aprica Müll.Hal.- Loc.: 5-WR-Özen 92.

*Philonotis caespitosa Jur. - Loc.: 2-WS-Özen 93.

P. fontana (Hedw.) Brid. - Loc.: 3-S-Özen 94, R-Özen 95, NS-Özen 96.

P. tomentella Molendo - Loc.: 3-S- Özen 97; 5R-Özen 98, NS-Özen 99.

Bryaceae Schwagr.

Ptychostomum capillare (Hedw.) Holyoak \& N. Pedersen- Loc.: 2-S-Özen 100, R-Özen 101.

P. moravicum (Podp.) Ros \& Mazimpaka- Loc.: 5-S-Özen 102, TB-Özen 103.
P. pseudotriquetrum (Hedw.) J.R. Spence \& H.P. Ramsay var. pseudotriquetrum - Loc.: 2NS-Özen 104, WS-Özen 105.

P. pseudotriquetrum var. bimum (Schreb.) Holyoak \& N. Pedersen- Loc.: 3-NS-Özen 106, WS-Öznur 107.

P. torquescens Bruch \& Schimp. - Loc.: 5-SÖzen 108, WS-Özen 109.

Mniaceae Schwagr.

Mnium hornum Hedw. - Loc.: 3-WS-Özen 110.

Plagiomnium affine (Blandow ex Funck) T.J.Kop. - Loc.: 1-WS-Özen 111; 5-WS-Özen 112.

P. elatum (Bruch \& Schimp.) T.J. Kop. - Loc.: 1-WS-Özen 113.

P. ellipticum (Brid.) T.J.Kop. - Loc.: 1-WSÖzen 114; 2-WS-Özen 115; 5-WS-Özen 116; 6WS-Özen 117.

P. medium (Bruch \& Schimp.) T.J.Kop. - Loc.: 5-WS-Özen 118.

P. rostratum (Schrad.) T.J.Kop. - Loc.: 2-WSÖzen 119.

P. undulatum (Hedw.) T.J.Kop. - Loc.: 1-S-Özen 120; 2-S-Özen 121; 5-R-Özen 122.

Pohlia annotina (Hedw.) Lindb. - Loc.: 6-SÖzen 123.

Cinclidiaceae Kindb.

Rhizomnium punctatum (Bruch \& Schimp.) T.J.Kop. - Loc.: 1-WS-Özen 124; 2-WS-Özen 125; 5-WS-Özen 126.

Orthotrichaceae Arn.

Ulota crispa (Hedw.) Brid. - Loc.: 3-TB-Özen 127.

Plagiotheciaceae (Broth.) M.Fleisch.

Plagiothecium cavifolium (Brid.) Z. Iwats.. Loc.: 1-WS-Özen 128; 5-WS-Özen 129.

P. succulentum (Wilson) Lindb.. - Loc.: 2-WSÖzen 130.

Amblystegiaceae Kindb.

Pseudoamblystegium subtile (Hedw.) Vanderp. \& Hedenäs - Loc.: 1-TB-Özen 131.

Thuidiaceae Schimp.

Abietinella abietina (Hedw.) M.Fleisch. var. abietina - Loc.: 2-S-Özen 132.

Thuidium assimile (Mitt.) A.Jaeger. - Loc.: 3WS-Özen 133.

T. delicatulum (Hedw.) Schimp. - Loc.: 1-WSÖzen 134; 3-WS-Özen 135.

*T. recognitum (Hedw.) Lindb. - Loc.: 6-WSÖzen 136.

T. tamariscinum (Hedw.) Schimp. - Loc.: 2-WSÖzen 137; 3-WS-Özen 138; 5-WS-Özen 139.

Brachytheciaceae Schimp.

Brachythecium albicans (Hedw.) Schimp. Loc.: 2-S-Özen 140; 3-S-Özen 141; 4-S-Özen 142; 5-S-Özen 143.

\#+B.capillaceum (F.Weber \& D.Mohr) Giacom. Loc: 1-S-Özen 144, R-Özen 145. 
B. rutabulum (Hedw.) Schimp. - Loc.: 1-WSÖzen 146; 2-WS-Özen 147; 3-NS-Özen 148; 5WS-Özen 149.

Eurhynchium striatum (Hedw.) Schimp. - Loc.: 1-R-Özen 150; 2-S-Özen 151; 3-R-Özen 152; 4S-Özen 153; 5-R-Özen 154.

Eurhynchiastrum pulchellum (Hedw.) Ignatov \& Huttunen- Loc.: 1-S-Özen 155, R-Özen 156.

Homalothecium aureum (Spruce) H.Robins. Loc.: 2-R-Özen 157.

Oxyrrhynchium hians (Hedw.) Loeske - Loc.: 5S-Özen 158, R-Özen 159.

Palamocladium euchloron (Müll.Hal.) Wijk \& Margad. - Loc.: 2-R-Özen 160.

Pseudoscleropodium purum (Hedw.) M.Fleisch. - Loc.: 3-S-Özen 161, R-Özen 162.

Rhynchostegium riparioides (Hedw.) CardoLoc.: 2-WR-Özen 163, NS-Özen 164.

$R$. rotundifolium (Scop. ex Brid.) Schimp. Loc.: 5-WR-Özen 165.

Sciuro-hypnum flotowianum (Sendtn.) Ignatov \& Huttunen - Loc.: 5-S-Özen 166.

Hypnaceae Schimp.

Herzogiella seligeri (Brid.) Z.Iwats. - Loc.: 4DTT-Özen 167.

Hypnum andoi A.J.E.Sm - Loc.: 2-R-Özen 168; 6-TB-Özen 169.

H. cupressiforme Hedw. - Loc.: 6-S-Özen 170, R-Özen 171.

*H. recurvatum (Lindb. \& Arnell) Kindb. - Loc.: 4-R-Özen 172.

Pylaisiaceae Schimp.

Calliergonella cuspidata (Hedw.) Loeske - Loc.: 3-WS-Özen 173, NS-Özen 174.

${ }^{*}$ C. lindbergii (Mitt.) Hedenäs - Loc.: 3-WSÖzen 175, NS-Özen 176.

Pylaisia polyantha (Hedw.) Schimp. - Loc.: 1TB-Özen 177; 6-TB-Özen 178.

Pylaisiadelphaceae Goffinet \& W. R. Buck

Platygyrium repens (Brid.) Schimp. - Loc.: 1TB-Özen 179.

Hylocomiaceae M. Fleisch.

Ctenidium molluscum (Hedw.) Mitt. - Loc.: 1-SÖzen 180; 2-S-Özen 181; 3-R-Özen 182; 4-SÖzen 183; 5-S-Özen 184; 6-R-Özen 185.

*Rhytidiadelphus squarrosus (Hedw.) Warnst. Loc.: 3-S-Özen 186.

Entodonceae Kindb.

Entodon concinnus (De Not.) Paris - Loc.: 2-SÖzen 187, R-Özen 188.

Neckeraceae Schimp.

Alleniella complanata (Hedw.) S.Olsson, Enroth \& D.Quandt - Loc.: 5-R-Özen 189, TB-Özen 190.

Exsertotheca crispa (Hedw.) S. Olsson, Enroth \& D. Quand - Loc.: 6-R-Özen 191, TB-Özen 192.
Thamnobryum alopecurum (Hedw.) Gangulee Loc.: 1-WS-Özen 193; 2-WR-Özen 194.

Lembophyllaceae Broth.

Isothecium alopecuroides (Lam. ex Dubois) Isov. - Loc.: 3-S-Özen 195; 5-TB-Özen 196; 6S-Özen 197.

I. myosuroides Brid. - Loc.: 1-R-Özen 198.

\section{Results and Discussion}

\subsection{Results}

As a result of the study, 18 liverwort taxa (belonging to 10 families and 12 genera), 81 moss taxa (belonging to 24 families and 50 genera) and a total of 99 bryophyte taxa (belonging to 34 families and 62 genera) were determined. The largest numbers of liverwort species were found in the family Scapaniaceae (4 taxa), Plagiochilaceae (3 taxa), Calypogeiaceae (2 taxa), Frullaniaceae (2 taxa), Pelliaceae (2 taxa). Finally, the others family, Radulaceae, Metzgeriaceae, Arnelliaceae, Jubulaceae, and Conocephaleaceae were represented by one taxon. In the case of mosses, they are represented by 50 genera and 81 taxa. The family Brachytheciaceae, having 12 mosses taxa, is the richest one, and followed by Mniaceae (8), Pottiaceae (7), Leucobryaceae (7), Bryaceae (5), Polytrichaceae (5), Thuidiaceae (5), Bartramiaceae (4), Hypnaceae (4), Grimmiaceae (3), Neckeraceae (3), Pylaisiaceae (3), Hylocomiaceae (2), Lembophyllaceae (2), Plagiotheciaceae (2). Other families of mosses (Amblystegiaceae, Cinclidiaceae, Orthotrichaceae, Dicranaceae, Dicranellaceae, Fissidentaceae, Rhabdoweisiaceae, Entodonceae, and Pylaisiadelphaceae) have only one taxon.

\subsection{Discussion}

We were determined 99 taxa (species, subspecies, and varieties) belonging to 34 families and 62 genera Bryophyta. All of these taxa were reported for the first time from Hayrat district (Trabzon). Brachythecium capillaceum (F.Weber \& D.Mohr) Giacom. was recorded as a new record for Trabzon Province and the second record for A4 grid-square. Southbya tophacea (Spruce) Spruce was reported for the second time from Trabzon and A4 grid-square. Also among 99 bryophyte taxa, 9 taxa (Campylopus brevipilus Bruch \& Schimp., $C$. flexuosus (Hedw.) Brid., C. pyriformis (Schultz) Brid., Bryoerythrophyllum ferruginascens (Stirt.) Giacom., Philonotis caespitosa Jur., Thuidium recognitum (Hedw.) Lindb., $H$. recurvatum (Lindb. \& Arnell) Kindb., Calliergonella lindbergii (Mitt.) Hedenäs, Rhytidiadelphus squarrosus (Hedw.) Warnst.) were reported for the second time from Trabzon. 
The most common genera of liverworts are Scapania (2), Calypogeia (2), Pellia (2), Diplophyllum (2), Plagiochila (2) and Frullania (2) in the study area. The most common genera of mosses are Plagiomnium (6), Ptychostomum (5), Campylopus (5), Thuidium (4), Philonotis (3), Brachythecium (3), Hypnum (3), Pogonatum (2), Polytrichum (2), Schistidium (2), Leucobryum (2), Tortella (2), Plagiothecium (2), Rhynchostegium (2), Calliergonella (2) and Isothecium (2) in the study area.

\section{References}

Abay G. Batan N. Özdemir T. 2016. Bryophyte checklist of Rize, north-east Turkey. Arctoa. 25: 386-392.

Akman Y. 1999. Climate and bioclimate. The methods of bioclimate and climate types of Turkey. Kariyer Matbaacılik. Ankara.

Atay S. Güleryüz G. Orhun C. Seçmen Ö. Vural C. 2009. Türkiye'nin 120 alpin bitkisi. Dönence Basım ve Yayın Hizmetleri, İstanbul.

Batan N. Özdemir T. 2011. Mersin (C12), Trabzon ve Gümüşhane (A4)' den bazı karayosunu (musci) kayıtları. SDÜ Orman Fakültesi Dergisi. 12:2, 104-109.

Batan N. Özdemir T. 2013. Bryoflora of Dernekpazarı District of Trabzon Province. Biological Diversity and Conservation. 6: 45-49.

Batan N. Alataş M. Özdemir T. 2013. Leptoscyphus cuneifolius (Lophocoleaceae, Marchantiophyta) new to Southwest Asia. Cryptogamie, Bryologie. 34: 373-377.

Blom H.H. 1996. A Revision of the Schistidium apocarpum Complex in Norway and Sweden, ISBN: 3-443-62021-3. Bryophytorum Bibliotheca.

Brugués M. Cros R.M. Guerra J. 2007. Flora Briofitica Ġbérica Volume I, Uniersidad de Murcia, ISBN: 978-84-611-8462-0. Sociedad Espanola de Briyologia Murcia. Murcia.

Frey W. Frahm J.P. Fischer E. Lobin W. 2006. The liverworts, mosses and ferns of Europe. Essex. Harley Books.

Gökler İ. 1998. Liverworts (Marchantiopsida) of the Altindere Valley National Park. Turkish Journal of Botany. 22: 409-412.

Guerra J. Cano M.J. Cros R.M. 2006. Flora Briofitica Ibérica VoIume 3, Uniersidad de Murcia, ISBN: 84-609-9097-4. Sociedad Espanola de Briyologia Murcia. Murcia.
Henderson D.M. 1961. Contribution to the Bryophyte Flora of Turkey: IV. Royal Botanic Garden Edinburgh. 23: 263-278.

Kırmac1 M. Kürschner H. Erdağ A. 2012. New and noteworthy records to the bryophyte flora of Turkey and Southwest Asia. Cryptogamie, Bryologie. 33: 267-270.

Kırmac1 M. Kürschner H. 2013. The genus Sphagnum L. in Turkey - with $S$. contortum, S. fallax, S. magellanicum and S. rubellum new to Turkey and Southwest Asia. Nova Hedwigia. 96: 383-397.

Kürschner H. Frey W. 2011. Liverworts, mosses and hornworts of Southwest Asia (Marchantiophyta, Bryophyta, Anthocerotophyta. Nova Hedwigia. 139: $1-240$.

Lara F. Mazımpaka V. Medina R. Caparros R. Garilleti R. 2010. The northeastern Turkey, an unnoticed but very important area for the Orthotrichaceae (Musci, Bryophyta). Nova Hedwigia. 138: 165180.

Nyholm E. 1986. Illustrated Flora of Nordic Mosses, Fasc. 1. FissidentaceaeSeligeriaceae. The Nordic Bryological Society. Lund.

Nyholm E. 1989. Illustrated Flora of Nordic Mosses, Fasc. 2. Pottiaceae Splachnaceae- Schistostegaceae, 75-141 pp. The Nordic Bryological Society. Lund.

Nyholm E. 1993. Illustrated Flora of Nordic Mosses, Fasc. 3. BryaceaeRhodobryaceae Mniaceae-CinclidiaceaePlagiomniaceae. 145-244 pp. The Nordic Bryological Society. Lund.

Nyholm E. 1998. Illustrated Flora of Nordic Mosses, Fasc. 4. AulacomniaceaeMeesiaceae-Catocopiaceae-

Bartramiaceae-Timmiaceae-

Encalyptaceae-Grimmiaceae-

Ptychomitriaceae-Hedwigiaceae-

Orthotrichaceae. 145-244 pp. The Nordic Bryological Society. Lund.

Özdemir T. Batan N. 2017a. The bryophyte checklist of Trabzon Province of Turkey. Arctoa. 26: 58-67.

Özdemir T. Batan N. 2017b. Bryophyte Checklist of Giresun, North East Turkey. Anatolian Bryology. 3:1, 1-8.

Papp B. 2004. Contributions to the bryoflora of the Pontic Mountains, North Anatolia, Turkey. Studia Botanica Hungarica. 35: 81-89.

Paton J. 1999. The Liverworts Flora of the British Isles, ISBN: 0-946589-60-7, 626 pp, Harley Books. England. 
Pedrotti C.C. 2001. Flora dei muschi d'Italia (Sphagnopsida, Andreaeopsida, Bryopsida, I parte). Antonio delfino Editore medicinascienze. Roma.

Pedrotti C.C. 2006. Flora dei muschi d'Italia. Bryopsida (II parte). Antonia Delfi no Editore medicina-scienze, Roma.

Ros R.M. Mazimpaka V. Abou-Salama U. Aleffi M. Blockeel T.L. Brugués M. Cros R.M. Dia M.G. Dirkse G.M. Draper I. et al., 2013. Mosses of the Mediterranean, an annotated checklist. Cryptogamie Bryologie. 34: 99-283.

Smith A.J.E. 1996. The Liverworts of Britain and Ireland, ISBN: 0-521-42473-9, 384 pp. Cambridge University Press. Cambridge.

Smith A.J.E. 2004. The Moss Flora of Britain and Ireland. Second Edition, ISBN: 052181640-8, 1012 pp. Cambridge University Press. Cambridge.

Söderström L. Hagborg A. Von Konrat M. Bartholomew-Began S. Bell D. Briscoe L. Brown E. Cargill D.C. Costa D.P. Barbara J. et al. 2016. World Checklist of Hornworts and Liverworts. PhytoKeys. 59: $1-828$.

Townsend C.C. 2005. Mosses from the Caucasian region and eastern Turkey. Journal of Bryology. 27: 143-152.

Vural M. 1996. Rize'nin yüksek dağ vejetasyonu. Turkish Journal of Botany. 20: 83-102. 